\title{
Group Decision-making Method Based on Grey Relational Analysis and Evidence Theory for XAI
}

\section{Decai Sun ( $\nabla$ decai0115@126.com )}

North China University of Water Resources and Electric Power

Dang Luo

North China University of Water Resources and Electric Power

\section{Research Article}

Keywords: Group decision making, Information fusion, Evidence theory, Comprehensive grey relational analysis, Explainable Al

Posted Date: June 7th, 2021

DOI: https://doi.org/10.21203/rs.3.rs-551277/v1

License: (1) This work is licensed under a Creative Commons Attribution 4.0 International License. Read Full License 


\title{
Group Decision-Making Method Based on Grey Relational Analysis and Evidence Theory for XAI
}

\author{
Decai Sun ${ }^{\mathrm{a} *}$, Dang Luo ${ }^{\mathrm{b}}$ \\ ${ }^{a}$ School of Management and Economics, North China University of Water Resources \\ and Electric Power, Zhengzhou 450046, China \\ ${ }^{b}$ School of Mathematics and Statistics, North China University of Water Resources \\ and Electric Power, Zhengzhou 450046, China
}

\begin{abstract}
For the uncertainty and complexity ingroup decision making and the differences of decision makers' reliabilities, a group decision making method based on grey relational analysis and evidence theory is proposed. Combining grey relational analysis with evidence theory, a novel decision-making method extracting the degree of ignorance for individual decision makers' information and constructing the Mass function is presented based on the comprehensive grey relational analysis (CGRA) method. We should also address how AI systems make their black box decisions, which calls for research into Explainable AI (XAI) by pursuing reverse engineering and self-explainability in AI. Considering the differences of decision makers' reliabilities, the Mass function is modified by the evidence weight, and the group decision information is fused by the Dempster's combination rule. On this basis, the Mass function is further transformed into the probability by the Pignistic probability transformation, which issued for ranking analysis of group decision making. Finally, the proposed method is applied to the green supplier selection, and the comparative analysis is further performed to verify the rationality and effectiveness of the proposed method.
\end{abstract}

Keywords: Group decision making; Information fusion; Evidence theory; 
Comprehensive grey relational analysis; Explainable AI

\section{Introduction}

Multi-attribute group decision making is widely used in economy (Tang et al. 2019), management (Cali and Balaman 2019), engineering (Peng 2019) and other fields because it can extract the cognitive judgment information of multiple decision makers and carry out a comprehensive evaluation to alternatives. In group decision making, how to effectively fuse the individual decision-making information is the key of decision-making analysis, which has an important influence on the ranking of alternatives.

In reality, when making group decision, besides the weighted sum of evaluation values of alternatives under group decision makers for ranking analysis, in more cases, it uses the individual experience and the collected relevant facts and data as evidence of judgment and reasoning, and then finds out the optimal alternative. Here, it is called the group decision making of evidence reasoning. Evidence theory (Chemoun et al. 2018), as an effective information fusion technology, has been widely used in uncertain reasoning, information fusion and group decision-making analysis. At present, some scholars have integrated evidence theory into group decision making, and discussed the group decision making methods of evidence reasoning. Wang et al. (2015) proposed a multi-objective group decision-making CBM model based on D-S evidence theory. Leung et al. (2013) combined group decision making with evidence theory and developed a comprehensive method for automatically identifying and eliminating unreliable evidence. Gupta et al. (2016) utilized evidence theory, Bayes approximation and VIKOR method to present a multi-attribute group decision-making method. Wan et al. (2013) proposed a multi-attribute group decision-making method, in which the decision makers' weights were determined by evidence theory and Bayes 
approximation. The introduction and application of these methods have promoted the further development of evidence theory and group decision making theory.

In group decision making, evidence theory can effectively fuse Mass functions of evidence bodies from individual decision makers. Because the quality of Mass function construction is directly related to whether the uncertainty characteristics of the problem can be described reasonably, and it has a decisive impact on the subsequent combination reasoning and group decision-making effect. Hence, accurately obtaining Mass functions is a key link in applying evidence theory (Zhou et al. 2018). At present, the determination of Mass function is generally a digital representation of support degree of different propositions in the frame of discernment based on the obtained evidence and personal experience, which has a certain subjectivity (Sun et al. 2018). In addition, different experts may give different Mass functions for the same proposition with the same evidence. Hence, how to determine the Mass function scientifically to reduce the group decision-making uncertainty is an urgent problem.

The Mass function of alternative under each individual decision maker is affected by the decision maker's reliability and its own measurement value, in which the measurement value can be reflected by the relational degree between alternative sequence and positive (negative) ideal point sequence. The greater the relational degree, the higher the event probability, and the larger the Mass function corresponding to the alternative. Grey relational analysis (GRA) is an effective method for describing the relational degree ( $\mathrm{Li}$ et al. 2015). Its basic idea is to judge the relational degree of factors according to the similarity degree of sequence curves, and it has the advantage of no special requirements for the sample size and statistical regularity (Wang and Yao 2018). Therefore, GRA can be adopted to construct Mass 
function objectively. However, the existing GRA focuses on measuring the proximity of geometric shapes between curves, while the similarity of speed and acceleration and the volatility of curve development trend are less considered. It is necessary to measure GRA degree more objectively and comprehensively.

In addition, when using evidence theory to solve group decision making problems, the reliability of evidence information of individual decisionmakers is usually no significant difference (Wang and Dang 2009). But in actual decision-making, the reliabilities of different decision makers are often different because of their professional background, social status, knowledge, and other reasons. Especially in the case of many alternatives and attributes, if decision makers' evaluation information is simply weighted average or the differences are ignored, it will inevitably affect the final evaluation result. Therefore, it is necessary to consider the differences of decision makers' reliabilities. However, the current research on group decision-making seldom considers the influence of the differences. If these differences are introduced into evidence reasoning and modified the evidence information, it will undoubtedly promote the effect of group decision information fusion.

According to the above analysis, starting from the idea of information fusion, the paper combines GRA with evidence theory, a method of extracting the degree of ignorance (DOI) for individual decision makers' information and constructing Mass function based on CGRA is proposed. Then, a weighted evidence theory is adopted to fuse the group decision information, and alternative ranking analysis is performed according to Pignistic probability transformation. Finally, the proposed method is used to deal with the problem of green supplier selection, and achieve a satisfactory result. 
The main structure of this paper is as follows: Section 2 starts with a brief presentation of necessary related concepts. Section 3 proposes a group decision making method of relational evidence reasoning based on information fusion. In Section 4 the proposed method is used to evaluate the green suppliers. Finally, some conclusions are presented in Section 5.

\section{Preliminaries}

\subsection{Evidence theory}

Evidence theory is an uncertain reasoning method, which can fuse data from different sources. It has been widely used in the fields of information fusion (Seiti and Hafezalkotob 2018), fault diagnosis (Lin et al. 2018). In evidence theory, $\Theta=\left\{g_{1}, g_{2}, \mathrm{~L}, g_{N}\right\}$ is the frame of discernment, and $g_{l} \mathrm{I} g_{q}=\varnothing(l \neq q)$. If there are $N$ elements in $\Theta$, the power set consisted by all subsets of $\Theta$ is $2^{\Theta}=\left\{\varnothing,\left\{g_{1}\right\}, \mathrm{L},\left\{g_{N}\right\},\left\{g_{1}, g_{2}\right\}, \mathrm{L},\left\{g_{1}, g_{N}\right\},\left\{g_{1}, g_{2}, g_{N}\right\}, \mathrm{L}, \Theta\right\}$.

Definition 1(Khojasteh and Jadid 2017) Let $E$ be any subset of $\Theta=\left\{g_{1}, g_{2}, \mathrm{~L}, g_{N}\right\}$, if there exists a mapping $m: 2^{\Theta} \rightarrow[0,1]$ which satisfies $m(\varnothing)=0, m(E) \in[0,1]$, $\sum_{E \subseteq \Theta} m(E)=1$, then $m(E)$ is called the basic probability assignment (BPA) function of $E$ on $\Theta$, also called Mass function. For any $E \subseteq \Theta, m(E)$ represents the precise degree of trust in $E$.

Definition 2For any $E \subseteq \Theta$, let $m_{1}, m_{2}, \mathrm{~L}, m_{n}$ be the Mass functions on $\Theta$, then $m_{1}, m_{2}, \mathrm{~L}, m_{n}$ can be combined with Dempster's combination ruleas follows

$$
\left(m_{1} \oplus m_{2} \oplus \mathrm{L} \oplus m_{n}\right)(E)=\left\{\begin{array}{l}
0, E=\varnothing ; \\
\frac{1}{K_{E_{1} \mathrm{I}}} \sum_{E_{2} \mathrm{I} \mathrm{L} \mathrm{I} E_{n}=E} m_{1}\left(E_{1}\right) m_{2}\left(E_{2}\right) \mathrm{L} m_{n}\left(E_{n}\right), E \neq \varnothing .
\end{array}\right.
$$


Where $K=\sum_{E_{1} \mathrm{I} \mathrm{L} \text { I } E_{n} \neq \varnothing} m_{1}\left(E_{1}\right) \cdot m_{2}\left(E_{2}\right) \mathrm{L} m_{n}\left(E_{n}\right) . K$ is a normalized coefficient, and the size of $K$ reflects the degree of conflict among evidence.

\subsection{Pignistic probability transformation}

Pignistic probability was first proposed by Smith to solve system decision problems with uncertain information. Smets further analyzed its theoretical basis, and proposed to convert Mass function into Pignistic probability function, which provided an effective solution to uncertain decision-making problems (Smets 2005).

Definition 3Let $m(G)$ be a Mass function on $\Theta$, then its Pignistic probability function $\operatorname{Bet}_{m}(\delta): \Theta \rightarrow[0,1]$ is defined as follows

$$
\operatorname{BetP}_{m}(\delta)=\sum_{G \subseteq \Theta, \delta \in G} \frac{1}{|G|} \frac{m(G)}{1-m(\varnothing)}
$$

Where $m(\varnothing) \neq 1,|G|$ is the cardinality of $\operatorname{subset} G$. Pignistic transformation is the process of converting $m$ to $\operatorname{BetP}_{m}$, which uses to make a decision. If no otherwise specified, it is considered that $m(\varnothing)=0$, and $\frac{m(G)}{1-m(\varnothing)}$ is simplified to $m(G)$.

\section{The proposed group decision making method}

In multi-attribute group decision making, let $A=\left\{a_{1}, a_{2}, \mathrm{~L}, a_{n}\right\}$ and $B=\left\{b_{1}, b_{2}, \mathrm{~L}, b_{m}\right\}$ be the alternative set and attribute set, respectively. $D M=\left\{D M_{1}, D M_{2}, \mathrm{~L}, D M_{h}\right\}$ is a set of decision makers, and $\psi_{k}\left(\psi_{k} \in[0,1]\right)$ is the weight of $D M_{k}(k=1,2, \mathrm{~L}, h)$, where $\sum_{k=1}^{h} \psi_{k}=1$. Let $c_{i j}^{(k)}$ be the attribute value provided by $D M_{k}$ on the assessment of $a_{i}$ about $b_{j}$, then the decision matrix of $D M_{k}$ is $\mathbf{C}^{(k)}=\left[c_{i j}^{(k)}\right]_{n \times m}$. The problem to be solved is how to effectively fuse group 
decision making information and use decision making analysis method to rank alternatives.

In order to eliminate the differences of attributes in dimension, let $c_{j}^{(k)^{-}}=\min _{i}\left\{c_{i j}^{(k)}\right\}, c_{j}^{(k)^{+}}=\max _{i}\left\{c_{i j}^{(k)}\right\}$, the decision matrix $\mathbf{C}^{(k)}=\left[c_{i j}^{(k)}\right]_{n \times m}$ can be normalized as $\mathbf{X}^{(k)}=\left[x_{i j}^{(k)}\right]_{n \times m}$, where

$$
x_{i j}^{(k)}=\left\{\begin{array}{l}
\frac{c_{i j}^{(k)}-c_{j}^{(k)^{-}}}{c_{j}^{(k)^{+}}-c_{j}^{(k)^{-}}}, \text {for the benefit attribute } b_{j} \\
\frac{c_{j}^{(k)^{+}}-c_{i j}^{(k)}}{c_{j}^{(k)^{+}}-c_{j}^{(k)^{-}}}, \text {for the cost attribute } b_{j}
\end{array}\right.
$$

In information fusion decision making, the key to aggregating group information with evidence theory is to obtain the Mass function of the alternative (also known as the evidence) under individual decisionmakers. However, the existing Mass function is usually set subjectively. To obtain the Mass function more objectively and reasonably, a CGRA method is proposed to determine the Mass functions of alternatives. On this basis, the group evidence information is fused by weighted evidence theory and the alternatives are ranked by Pignistic probability transformation.

\subsection{Construction of Mass Function Based on CGRA Method}

Since the Mass functions under individual decision makers are the key to synthesizing group information with evidence theory, the premise of solving Mass function is to determine the degree of ignorance (DOI)for individual decision makers' information (Wang and Dang 2009). Hence, combining GRA with evidence theory, a CGRA method is proposed to determine the DOI and the Mass function. 


\subsubsection{The proposed CGRA method}

To measure the correlation accurately, the proximity, similarity and volatility of sequence evolution are comprehensively measured by considering the absolute level, dynamic level and variability level among sequences. On this basis, the comprehensive grey relational analysis (CGRA) method is constructed.

For the proximity between the alternative sequence and the positive (negative) ideal point sequence, their absolute level can be measured by the overall displacement change between the sequences.

Definition 4 Let $a_{i}^{(k)}=\left(x_{i 1}^{(k)}, x_{i 2}^{(k)}, \mathrm{L}, x_{i m}^{(k)}\right)(i=1,2, \mathrm{~L}, n)$ and $a^{(k)^{+(-)}}=\left(x_{1}^{(k)^{+(-)}}, x_{2}^{(k)^{+(-)}}, \mathrm{L}, x_{m}^{(k)^{+(-)}}\right)$be the alternative sequence and the positive (negative) ideal point sequence under decision maker $D M_{k}$ respectively, then the overall displacement change of $a_{i}^{(k)}$ and $a^{(k)^{+(-)}}$is expressed as follows

$$
d_{i}^{(k)^{+(-)}}=\left[\sum_{j=1}^{m}\left(x_{i j}^{(k)}-x_{j}^{(k)^{+(-)}}\right)^{2}\right]^{1 / 2}
$$

Where $x_{j}^{(k)^{+}}=\max _{1 \leq i \leq n} x_{i j}^{(k)}, x_{j}^{(k)^{-}}=\min _{1 \leq i \leq n} x_{i j}^{(k)} \cdot d_{i}^{(k)^{+(-)}}$characterizes the proximity of the absolute level between $a_{i}^{(k)}$ and $a^{(k)^{+(-)}}$in development process, the positive ideal point sequence corresponds to the superscript + , and the negative ideal point sequence corresponds to the superscript -. When $d_{i}^{(k)^{+(-)}}=0$, it indicates that the development process of $a_{i}^{(k)}$ and $a^{(k)^{+(-)}}$is exactly the same.

To improve the distinguishing degree of relational results in traditional GRA method, the proximity relational degree is constructed based on Liu et al. (2014), which adopts the form of exponential function $f(x)=e^{-x}$ with faster decline rate and stronger distinguishability. 
Definition 5 Let $d_{i}^{(k)^{+(-)}}$be the overall displacement change of sequences $a_{i}^{(k)}$ and $a^{(k)^{+(-)}}$under decision maker $D M_{k}$, then the proximity relational degree of $a_{i}^{(k)}$ and $a^{(k)^{+(-)}}$is expressed as follows

$$
\alpha_{i}^{(k)^{+(-)}}=\exp \left(-d_{i}^{(k)^{+(-)}}\right)
$$

Where $\alpha_{i}^{(k)^{+(-)}}$measures the proximity degree of absolute amount between $a_{i}^{(k)}$ and $a^{(k)^{+(-)}}$from the perspective of proximity. The larger the $\alpha_{i}^{(k)^{+(-)}}$is, the closer the change of overall absolute level between $a_{i}^{(k)}$ and $a^{(k)^{+(-)}}$in development process will be.

For the similarity of development trends between the alternative sequence $a_{i}^{(k)}$ and the positive (negative) ideal point sequence $a^{(k)^{+(-)}}$, the dynamic level can be measured by the speed and acceleration of development trends of sequences.

Definition 6 For the alternative sequence $a_{i}^{(k)}$ and the positive (negative) ideal point sequence $a^{(k)^{+(-)}}$under decision maker $D M_{k}$, the similar speed relational coefficient of $a_{i}^{(k)}$ and $a^{(k)^{+(-)}}$from attribute $b_{j-1}$ to attribute $b_{j}$ is expressed as follows

$$
\beta_{i j}^{(k)^{+(-)}}=\operatorname{sgn}\left(\left(x_{i j}^{(k)}\right)^{\prime} \times\left(x_{j}^{(k)^{+(-)}}\right)^{\prime}\right) \cdot \frac{1+\left|\left(x_{i j}^{(k)}\right)^{\prime}\right|+\left|\left(x_{j}^{(k)^{+(-)}}\right)^{\prime}\right|}{1+\left|\left(x_{i j}^{(k)}\right)^{\prime}\right|+\left|\left(x_{j}^{(k)^{+(-)}}\right)^{\prime}\right|+\left|\left(x_{i j}^{(k)}\right)^{\prime}-\left(x_{j}^{(k)^{+(-)}}\right)^{\prime}\right|}
$$

Where $\left(x_{i j}^{(k)}\right)^{\prime}$ and $\left(x_{j}^{(k)^{+(-)}}\right)^{\prime}$ are the speeds of sequences $a_{i}^{(k)}$ and $a^{(k)^{+(-)}}$from $b_{j-1}$ to $\quad b_{j}, \quad$ respectively; $\quad\left(x_{i j}^{(k)}\right)^{\prime}=\frac{x_{i j}^{(k)}-x_{i(j-1)}^{(k)}}{j-(j-1)}=x_{i j}^{(k)}-x_{i(j-1)}^{(k)} ; j=2,3, \mathrm{~L}, m$. Similarly, $\left(x_{j}^{(k)^{+(-)}}\right)^{\prime}=x_{j}^{(k)^{+(-)}}-x_{j-1}^{(k)^{+(-)}} ; \operatorname{sgn}\left(\left(x_{i j}^{(k)}\right)^{\prime} \times\left(x_{j}^{(k)^{+(-)}}\right)^{\prime}\right)=\left\{\begin{array}{l}1,\left(\left(x_{i j}^{(k)}\right)^{\prime} \times\left(x_{j}^{(k)^{+(-)}}\right)^{\prime}\right) \geq 0 \\ -1,\left(\left(x_{i j}^{(k)}\right)^{\prime} \times\left(x_{j}^{(k)^{+(-)}}\right)^{\prime}\right)<0\end{array}\right.$. 
According to the similar speed relational coefficient $\hat{\beta}_{i j}^{(k)^{+(-)}}$, the similar speed relational degree of $a_{i}^{(k)}$ and $a^{(k)^{+(-)}}$in the whole attribute set is expressed as follows

$$
\hat{\beta}_{i}^{(k)^{+(-)}}=\frac{1}{(m-1)} \sum_{j=2}^{m} \hat{\beta}_{i j}^{(k)^{+(-)}}
$$

Where $\hat{\beta}_{i}^{(k)^{+(-)}}$measures the similarity degree of development speed between $a_{i}^{(k)}$ and $a^{(k)^{+(-)}}$from the perspective of similarity. The larger the $\hat{\beta}_{i}^{(k)^{+(-)}}$is, the more similar the overall development speed between $a_{i}^{(k)}$ and $a^{(k)^{+(-)}}$will be.

Definition 7 For the alternative sequence $a_{i}^{(k)}$ and the positive (negative) ideal point sequence $a^{(k)^{+(-)}}$under decision maker $D M_{k}$, the similar acceleration relational coefficient of $a_{i}^{(k)}$ and $a^{(k)^{+(-)}}$from attribute $b_{j-1}$ to attribute $b_{j}$ is expressed as follows

$$
\hat{\beta}_{i j}^{(k)^{+(-)}}=\operatorname{sgn}\left(\left(x_{i j}^{(k)}\right)^{\prime \prime} \times\left(x_{j}^{(k)^{+(-)}}\right)^{\prime \prime}\right) \cdot \frac{1+\left|\left(x_{i j}^{(k)}\right)^{\prime \prime}\right|+\left|\left(x_{j}^{(k)^{+(-)}}\right)^{\prime \prime}\right|}{1+\left|\left(x_{i j}^{(k)}\right)^{\prime \prime}\right|+\left|\left(x_{j}^{(k)^{+(-)}}\right)^{\prime \prime}\right|+\left|\left(x_{i j}^{(k)}\right)^{\prime \prime}-\left(x_{j}^{(k)^{+(-)}}\right)^{\prime \prime}\right|}
$$

Where $\left(x_{i j}^{(k)}\right)^{\prime \prime}$ and $\left(x_{j}^{(k)^{+(-)}}\right)^{\prime \prime}$ are the accelerations of sequences $a_{i}^{(k)}$ and $a^{(k)^{+(-)}}$ from $b_{j-1}$ to $b_{j}$, respectively; $\left(x_{i j}^{(k)}\right)^{\prime \prime}=\frac{\left(x_{i j}^{(k)}\right)^{\prime}-\left(x_{i(j-1)}^{(k)}\right)^{\prime}}{2}=\frac{x_{i j}^{(k)}-2 x_{i(j-1)}^{(k)}+x_{i(j-2)}^{(k)}}{2}$; $j=3,4, \mathrm{~L}, m \quad$ Similarly, $\quad\left(x_{j}^{(k)^{+(-)}}\right)^{\prime \prime}=\frac{x_{j}^{(k)^{+(-)}}-2 x_{(j-1)}^{(k)^{+(-)}}+x_{(j-2)}^{(k)^{+(-)}}}{2}$ $\operatorname{sgn}\left(\left(x_{i j}^{(k)}\right)^{\prime \prime} \times\left(x_{j}^{(k)^{+(-)}}\right)^{\prime \prime}\right)=\left\{\begin{array}{l}1,\left(x_{i j}^{(k)}\right)^{\prime \prime} \times\left(x_{j}^{(k)^{+(-)}}\right)^{\prime \prime} \geq 0 \\ -1,\left(x_{i j}^{(k)}\right)^{\prime \prime} \times\left(x_{j}^{(k)^{+(-)}}\right)^{\prime \prime}<0\end{array}\right.$.

According to the similar acceleration relational coefficient $\hat{\beta}_{i j}^{(k)^{+(-)}}$, the similar acceleration relational degree of $a_{i}^{(k)}$ and $a^{(k)^{+(-)}}$in the whole attribute set is 
expressed as follows

$$
\hat{\beta}_{i}^{(k)^{+(-)}}=\frac{1}{m-2} \sum_{j=3}^{m} \hat{\beta}_{i j}^{(k)^{+(-)}}
$$

Where $\hat{\beta}_{i}^{(k)^{+(-)}}$measures the similarity degree of the change of development speed between $a_{i}^{(k)}$ and $a^{(k)^{+(-)}}$from the perspective of similarity. The larger the $\grave{\beta}_{i}^{(k)^{+(-)}}$is, the more similar the level of the change of overall development speed between $a_{i}^{(k)}$ and $a^{(k)^{+(-)}}$will be.

Definition 8 Let $\hat{\beta}_{i}^{(k)^{+(-)}}$and $\hat{\beta}_{i}^{(k)^{+(-)}}$be respectively the similar speed relational degree and acceleration relational degree of sequences $a_{i}^{(k)}$ and $a^{(k)^{+(-)}}$under decision maker $D M_{k}$, then the similarity relational degree of $a_{i}^{(k)}$ and $a^{(k)^{+(-)}}$is expressed as follows

$$
\beta_{i}^{(k)^{+(-)}}=\frac{1}{2}\left(\hat{\beta}_{i}^{(k)^{+(-)}}+\hat{\beta}_{i}^{(k)^{+(-)}}\right)
$$

Where $\beta_{i}^{(k)^{+(-)}}$measures the similarity degree of the development speed and the change of development speed between $a_{i}^{(k)}$ and $a^{(k)^{+(-)}}$from the perspective of similarity. The larger the $\beta_{i}^{(k)^{+(-)}}$is, the more similar the overall development speed and the level of the change of overall development speed between $a_{i}^{(k)}$ and $a^{(k)^{+(-)}}$ will be.

For the volatility of development trends between the alternative sequence $a_{i}^{(k)}$ and the positive (negative) ideal point sequence $a^{(k)^{+(-)}}$, the variability level can be measured by the change of volatility distance between sequences.

Definition 9 For the alternative sequence $a_{i}^{(k)}$ and the positive (negative) ideal point 
sequence $a^{(k)^{+(-)}}$under decision maker $D M_{k}$, the overall volatility distance of $a_{i}^{(k)}$ and $a^{(k)^{+(-)}}$is expressed as follows

$$
f_{i}^{(k)^{+(-)}}=\left|\frac{\sigma_{i}^{(k)}}{\bar{x}_{i}^{(k)}}-\frac{\sigma^{(k)^{+(-)}}}{\bar{x}^{(k)^{+(-)}}}\right|
$$

Where $\bar{x}_{i}^{(k)}=\frac{1}{m} \sum_{j=1}^{m} x_{i j}^{(k)}, \quad \sigma_{i}^{(k)}=\sqrt{\frac{1}{m-1} \sum_{j=1}^{m}\left(x_{i j}^{(k)}-\bar{x}_{i}^{(k)}\right)^{2}} \cdot \bar{x}_{i}^{(k)}$ and $\sigma_{i}^{(k)}$ denote the mean value and the standard deviation of variables about alternative $a_{i}$ in attribute set $B$, respectively. The meanings of $\bar{x}^{(k)^{+(-)}}$and $\sigma^{(k)^{+(-)}}$are similar to those of $\bar{x}_{i}^{(k)}$ and $\sigma_{i}^{(k)} \cdot f_{i}^{(k)^{+(-)}}$depicts the volatility difference between sequences $a_{i}^{(k)}$ and $a^{(k)^{+(-)}}$in development process. Obviously, if the proximity and similarity of the two sequences are greater, the degree of volatility should be similar. That is, $f_{i}^{(k)^{+(-)}}$should be smaller. Coefficient of variation is a representative of the degree of sequence volatility. Considering that the larger the coefficient of variation is, the bigger the degree of sequence volatility is, and the more unstable the development of sequence is. It can be seen that it is a negative index, so the reciprocal is used in calculating distance.

Definition 10 Let $f_{i}^{(k)^{+(-)}}$be the overall volatility distance of sequences $a_{i}^{(k)}$ and $a^{(k)^{+(-)}}$under decision maker $D M_{k}$, then the volatility relational degree of $a_{i}^{(k)}$ and $a^{(k)^{+(-)}}$is expressed as follows

$$
\gamma_{i}^{(k)^{+(-)}}=\exp \left(-f_{i}^{(k)^{+(-)}}\right)
$$

Where $\gamma_{i}^{(k)^{+(-)}}$measures the volatility degree of development trends between $a_{i}^{(k)}$ and $a^{(k)^{+(-)}}$from the perspective of volatility. The larger the $\gamma_{i}^{(k)^{+(-)}}$is, the more consistent the volatility degree between $a_{i}^{(k)}$ and $a^{(k)^{+(-)}}$in development process will 
be.

According to the definitions of proximity relational degree $\alpha_{i}^{(k)^{+(-)}}$, similarity relational degree $\beta_{i}^{(k)^{+(-)}}$and volatility relational degree $\gamma_{i}^{(k)^{+(-)}}$of $a_{i}^{(k)}$ and $a^{(k)^{+(-)}}$ under decision maker $D M_{k}$, the larger the $\alpha_{i}^{(k)^{+}}, \beta_{i}^{(k)^{+}}$and $\gamma_{i}^{(k)^{+}}$are, the closer the alternative $a_{i}$ is to the positive ideal alternative; the smaller the $\alpha_{i}^{(k)-}, \beta_{i}^{(k)-}$ and $\gamma_{i}^{(k)-}$ are, the further the alternative $a_{i}$ is from the negative ideal alternative. Based on this, the comprehensive grey relational analysis (CGRA) degree is constructed to measure the superiority and inferiority level between alternative and ideal alternatives under individual decision makers, which lays a foundation for further obtaining the Mass function of alternative (or call it evidence) under individual decision makers.

Definition 11 For the proximity relational degree $\alpha_{i}^{(k)^{+(-)}}$, similarity relational degree $\beta_{i}^{(k)^{+(-)}}$and volatility relational degree $\gamma_{i}^{(k)^{+(-)}}$of $a_{i}^{(k)}$ and $a^{(k)^{+(-)}}$under decision maker $D M_{k}$, the CGRA degree of alternative $a_{i}$ is expressed as follows

$$
\phi_{i}^{(k)}=w_{1} \frac{\alpha_{i}^{(k)^{+}}}{\alpha_{i}^{(k)^{+}}+\alpha_{i}^{(k)^{-}}}+w_{2} \frac{\beta_{i}^{(k)^{+}}}{\beta_{i}^{(k)^{+}}+\beta_{i}^{(k)^{-}}}+w_{3} \frac{\gamma_{i}^{(k)^{+}}}{\gamma_{i}^{(k)^{+}}+\gamma_{i}^{(k)^{-}}}
$$

Where $w_{1}, w_{2}$ and $w_{3}$ are respectively the weights of three kinds of relational degrees $\alpha_{i}^{(k)^{+(-)}}, \beta_{i}^{(k)^{+(-)}}$and $\gamma_{i}^{(k)^{+(-)}}$, and satisfies $w_{1}+w_{2}+w_{3}=1$. The weight coefficient $w_{v}(v=1,2,3)$ can be determined subjectively or objectively according to the actual situation of the study. The bigger $\phi_{i}^{(k)}$ is, the better alternative $a_{i}$ is under decision maker $D M_{k}$.

\subsubsection{Determination of Mass Function using CGRA Method}

On the basis of the proposed CGRA method in Section 3.1.1, the degree of 
ignorance (DOI) for individual decision makers' information and the Mass function can be obtained. The detailed contents are presented as follows.

If the distribution of alternatives under a decision maker $D M_{k}(k=1,2, \mathrm{~L}, h)$ is more concentrated on a certain alternative, it shows that the decision-making environment under $D M_{k}$ is more conducive to decision making. This is reflected in evidence theory, which indicates that the information DOI of $D M_{k}$ is lower, and the alternative under $D M_{k}$ should give a bigger Mass value than that of a decision maker with high DOI.

According to Definition 11, the CGRA degree is a measure of superiority and inferiority level among alternatives and ideal alternatives in individual decision-making environments. Based on this, we can obtain the following definitions.

Definition 12 For the CGRA degree $\phi_{i}^{(k)}$ of alternative $a_{i}$ under decision maker $D M_{k}(k=1,2, \mathrm{~L}, h)$, the information DOI of $D M_{k}$ is expressed as follows

$$
\operatorname{DOI}\left(D M_{k}\right)=\left(\prod_{i=1}^{n} \phi_{i}^{(k)}\right)^{\frac{1}{n}}
$$

When using evidence theory to make fusion decision on group information, it is necessary to fuse the evidence information of individual decision makers in order to choose the ideal alternative among many alternatives. The determination of Mass function is the basis of information fusion. Based on the above analysis, the Mass function $m_{i}^{(k)}$ of alternative $a_{i}$ under $D M_{k}$ is expressed as follows

$$
m_{i}^{(k)}=\left(1-D O I\left(D M_{k}\right)\right) \phi_{i}^{(k)}
$$

Due to the complexities of objective things and the limitations of human cognition, there is uncertainty in the overall understanding, the sum of Mass functions 
of all the alternatives under $D M_{k}$ is less than 1 , that is, $\sum_{i=1}^{n} m_{i}^{(k)}<1$. In order to make full use of the obtained information and reduce the uncertainty in the decision-making, the remaining part of Mass function is assigned to the frame of discernment $\Theta$ itself, which indicates the degree of support for all the alternatives and participates in the calculation of information fusion. Therefore, the Mass function of the overall uncertainty under $D M_{k}$ can be obtained as follows

$$
m_{\Theta}^{(k)}=1-\sum_{i=1}^{n} m_{i}^{(k)}
$$

\subsection{Fusion of Group Decision-making Information Based on Weighted Evidence}

\section{Theory}

In group decision-making, evidence theory usually treats the evidence information under individual decision makers equally. However, due to the differences of individual decision makers' own conditions and knowledge experience in practical application, the reliability of evidence provided by different individuals is often different, and the evidence information should be discounted according to the different reliabilities. Based on this, a modification method of evidence information based on evidence weight is proposed, and then the group decision-making information is fused.

\subsubsection{Modification of Mass Function Based on Evidence Weight}

Generally, the decision-making evidence under different individuals in system should have different weights. If the decision-making evidence under an individual is with the greatest comprehensive support or importance, the evidence information under the individual is more credible and the reliability is greater, and the impact on the final fusion conclusion is greater. 
Definition 13 Let the frame of discernment $\Theta$ have $u$ pieces of evidence $e_{1}, e_{2}, \mathrm{~L}, e_{u}$, and the corresponding Mass functions are $m_{1}, m_{2}, \mathrm{~L}, m_{u}, \eta\left(m_{s}\right)$ is the weight of evidence $e_{s}$, let $\eta\left(m_{t}\right)=\max _{1 \leq s \leq u}\left\{\eta\left(m_{s}\right)\right\}$, then $e_{t}$ is called the key evidence, and the reliability of $e_{s}$ about $e_{t}$ is expressed as follows

$$
\vartheta_{s}=\frac{\eta\left(m_{s}\right)}{\eta\left(m_{t}\right)}(s=1,2, \mathrm{~L}, u)
$$

According to the correlation algorithm of evidence weight, the Mass functions are modified and obtained as follows

$$
\left\{\begin{array}{l}
\hat{m}_{s}(F)=\vartheta_{s} m_{s}(F) \\
\hat{m}_{s}(\Theta)=1-\sum_{s=1}^{u} \hat{m}_{s}(F) \\
\forall F \in 2^{\Theta}, F \neq \Theta
\end{array}\right.
$$

\subsubsection{Synthesis of Evidence information}

After obtaining the evidence information under individual decision makers, Dempster's combination rule is used to fuse it to obtain the integrated Mass function, and then to realize the subsequent reasoning decision-making. The specific synthesis process is as follows.

Firstly, according to the Mass function $m_{i}^{(k)}$ of alternative $a_{i}(i=1,2, \mathrm{~L}, n)$ under decision maker $D M_{k}(k=1,2, \mathrm{~L}, h)$, the modified Mass functions of $a_{i}$ and the overall uncertainty are obtained by using the evidence weight, respectively;

$$
\begin{gathered}
\hat{m}_{i}^{(k)}=\vartheta_{k} m_{i}^{(k)} \\
\hat{m}_{\Theta}^{(k)}=1-\sum_{i=1}^{n} \hat{m}_{i}^{(k)}
\end{gathered}
$$

Where $\vartheta_{k}=\frac{\psi_{k}}{\max _{1 \leq k \leq h}\left(\psi_{k}\right)}$ is the reliability of evidence information of $D M_{k}$, 
$\psi_{k}\left(\psi_{k} \in[0,1]\right)$ is the weight of $D M_{k}$.

Then, by using Dempster's combination rule to fuse evidence information under individual decision makers, the integrated Mass functions of $a_{i}$ and the overall uncertainty are obtained, denoted as $m\left(a_{i}\right)$ and $m(\Theta)$, respectively.

\subsection{Optimization Decision-making of Alternatives Based on Pignistic Probability} Value

When using evidence theory for group decision-making analysis, we often need to convert the Mass function into a probability. That is to say, the Mass assignments on subsets are aggregated into a single element. As can be seen from Definition 3, the Pignistic probability transformation is based on average distribution, and the information loss is smaller during transformation. Hence, on the basis of considering the overall uncertainty of the system, the Pignistic probability function $\operatorname{Bet} P\left(a_{i}\right)$ of alternative $a_{i}$ is obtained as follows

$$
\operatorname{BetP}\left(a_{i}\right)=m\left(a_{i}\right)+\frac{m(\Theta)}{n}
$$

Where $m\left(a_{i}\right)$ and $m(\Theta)$ are the integrated Mass functions of $a_{i}$ and the overall uncertainty, respectively. $n$ is the number of alternatives. The alternatives can be ranked according to the Pignistic probability values. The larger the $\operatorname{Bet} P\left(a_{i}\right)$, the better the alternative $a_{i}$ will be.

\subsection{The steps of the proposed group decision-making method}

Step 1: Normalize decision matrices of individual decision makers with Equation (3);

Step 2: Calculate the CGRA degree of each alternative under individual decision makers with Equations (4)-(13); 
Step 3: Construct the information DOI of individual decision makers with Equation (14), then construct the Mass functions of the alternatives and the overall uncertainty under individual decision makers with Equations (15)-(16);

Step 4: Calculate the integrated Mass functions of the alternatives and the overall uncertainty according to Equations (17)-(18) and Dempster's combination rule;

Step 5: Calculate the Pignistic probability value of each alternative with Equation (19);

Step 6: Rank alternatives. Sorting the Pignistic probability values in a descending sequence. The larger the value, the better the performance of the corresponding alternative.

\section{Case study}

\subsection{Case background}

With the improvement of public environmental protection consciousness, enterprises pay more attention to green practice and green strategy. Green supply chain is just to alleviate the pressure on resources and environment with economic development. In the process of implementing green supply chain, a key link is to select the appropriate green supplier. The green supplier selection needs the joint participation of quality, procurement, logistics and other departments, and the influence of many evaluation attributes should also be considered. Its essence is the sorting process of multi-attribute group decision-making problems. The following is an example of green supplier selection for a product component to illustrate the practicality of the proposed method.

A manufacturer plans to select the best supplier for production from the initially identified four suppliers $\left(a_{1}, a_{2}, a_{3}, a_{4}\right)$, which are evaluated by the decision-making 
group consisting of quality department $\left(D M_{1}\right)$, procurement department $\left(D M_{2}\right)$ and logistics department $\left(D M_{3}\right)$. The literature of green supplier selection is reviewed. Additionally, the enterprise investigation and expert consultation are performed. Then the attribute system of green supplier selection is established, which includes 5 criteria and 12 attributes, as shown in Table 1.The decision-making evaluation information provided by decision-making group $\left(D M_{1}, D M_{2}, D M_{3}\right)$ is shown in Table 2, in which the attribute values of each supplier about attributes $b_{1}, b_{3}, b_{6}, b_{8}, b_{10}, b_{12}$ are given subjectively, and the attribute values about attributes $b_{2}, b_{4}, b_{5}, b_{7}, b_{9}, b_{11}$ are obtained by objective statistics.

Table 1 The attribute system of the green supplier selection

\begin{tabular}{|c|c|c|c|c|}
\hline Criterion layer & Attribute layer & Reference & Unit & Explanation \\
\hline \multirow{3}{*}{ Green ecology $\left(B_{1}\right)$} & $\begin{array}{l}\text { Green design of products } \\
\left(b_{1}\right)\end{array}$ & $\begin{array}{l}\text { Qin et al. } \\
2017\end{array}$ & Score & $\begin{array}{l}\text { Environmental pollution degree throughout the life } \\
\text { cycle }\end{array}$ \\
\hline & Resources recovery $\left(b_{2}\right)$ & $\begin{array}{l}\text { Tsui and } \\
\text { Wen } 2014\end{array}$ & $\%$ & Rate of recovery to input \\
\hline & $\begin{array}{l}\text { Carbon reduction } \\
\text { measures }\left(b_{3}\right)\end{array}$ & $\begin{array}{l}\text { Luthra et al. } \\
2017\end{array}$ & Score & Implementation of carbon reduction measures \\
\hline \multirow{2}{*}{$\begin{array}{l}\text { Product information } \\
\left(B_{2}\right)\end{array}$} & Product quality $\left(b_{4}\right)$ & $\begin{array}{l}\text { Roshandel } \\
\text { et al. } 2013\end{array}$ & $\%$ & The product qualified rate \\
\hline & Product price $\left(b_{5}\right)$ & $\begin{array}{l}\text { Govindan et } \\
\text { al. } 2017\end{array}$ & Yuan & The price of purchased product \\
\hline \multirow{3}{*}{$\begin{array}{l}\text { Enterprise } \\
\text { competitiveness }\left(B_{3}\right)\end{array}$} & Financial situation $\left(b_{6}\right)$ & $\begin{array}{l}\text { Lin et al. } \\
2015\end{array}$ & Score & Sources and distribution of operating funds \\
\hline & Market share $\left(b_{7}\right)$ & $\begin{array}{l}\text { Hamdan } \\
\text { and } \\
\text { Cheaitou } \\
2017\end{array}$ & $\%$ & $\begin{array}{l}\text { Proportion of the enterprise sales volume in market like } \\
\text { products }\end{array}$ \\
\hline & Technical level $\left(b_{8}\right)$ & $\begin{array}{l}\text { Govindan et } \\
\text { al. } 2014\end{array}$ & Score & The ability to support technical innovation \\
\hline \multirow{2}{*}{$\begin{array}{l}\text { Service capability } \\
\left(B_{4}\right)\end{array}$} & On time delivery $\left(b_{9}\right)$ & $\begin{array}{l}\text { Wu and } \\
\text { Blackhurst } \\
2009\end{array}$ & $\%$ & $\begin{array}{l}\text { The proportion of the number of punctual deliveries to } \\
\text { the number of total deliveries in a given time interval }\end{array}$ \\
\hline & $\begin{array}{l}\text { After-sale service level } \\
\left(b_{10}\right)\end{array}$ & $\begin{array}{l}\text { Amin and } \\
\text { Zhang } 2012\end{array}$ & Score & Customer satisfaction with after-sale service \\
\hline \multirow{2}{*}{$\begin{array}{l}\text { Innovative ability } \\
\left(B_{5}\right)\end{array}$} & $\begin{array}{l}\text { New product exploitation } \\
\text { ratio }\left(b_{11}\right)\end{array}$ & $\begin{array}{l}\text { Azadi et al. } \\
2015\end{array}$ & $\%$ & Ratio of new product value to total product value \\
\hline & Innovation input $\left(b_{12}\right)$ & $\begin{array}{l}\text { Mafakheri } \\
\text { et al. } 2011\end{array}$ & Score & $\begin{array}{l}\text { Investments of innovative personnel, equipment and } \\
\text { fund }\end{array}$ \\
\hline
\end{tabular}

Table 2 The decision matrices provided by $D M_{1}, D M_{2}, D M_{3}$

\begin{tabular}{|c|c|c|c|c|c|c|c|c|c|c|c|c|}
\hline \multirow{2}{*}{ Attribute } & \multicolumn{4}{|c|}{$D M_{1}$} & \multicolumn{4}{|c|}{$\mathrm{DM}_{2}$} & \multicolumn{4}{|c|}{$\mathrm{DM}_{3}$} \\
\hline & $a_{1}$ & $a_{2}$ & $a_{3}$ & $a_{4}$ & $a_{1}$ & $a_{2}$ & $a_{3}$ & $a_{4}$ & $a_{1}$ & $a_{2}$ & $a_{3}$ & $a_{4}$ \\
\hline$b_{1}$ & 0.20 & 0.23 & 0.21 & 0.30 & 0.24 & 0.15 & 0.19 & 0.28 & 0.27 & 0.21 & 0.18 & 0.32 \\
\hline$b_{2}$ & 35 & 38 & 36 & 31 & 35 & 38 & 36 & 31 & 35 & 38 & 36 & 31 \\
\hline$b_{3}$ & 0.81 & 0.84 & 0.82 & 0.78 & 0.75 & 0.87 & 0.83 & 0.80 & 0.81 & 0.85 & 0.88 & 0.82 \\
\hline$b_{4}$ & 89 & 96 & 92 & 84 & 89 & 96 & 92 & 84 & 89 & 96 & 92 & 84 \\
\hline
\end{tabular}




\begin{tabular}{ccccccccccccc}
$b_{5}$ & 26 & 25 & 24 & 28 & 26 & 25 & 24 & 28 & 26 & 25 & 24 & 28 \\
$b_{6}$ & 0.91 & 0.94 & 0.92 & 0.88 & 0.90 & 0.97 & 0.94 & 0.87 & 0.94 & 0.96 & 0.97 & 0.95 \\
$b_{7}$ & 15 & 17 & 14 & 12 & 15 & 17 & 14 & 12 & 15 & 17 & 14 & 12 \\
$b_{8}$ & 0.94 & 0.92 & 0.95 & 0.90 & 0.90 & 0.94 & 0.92 & 0.87 & 0.90 & 0.96 & 0.93 & 0.91 \\
$b_{9}$ & 91 & 96 & 97 & 92 & 91 & 96 & 97 & 92 & 91 & 96 & 97 & 92 \\
$b_{10}$ & 0.93 & 0.95 & 0.98 & 0.94 & 0.91 & 0.97 & 0.94 & 0.92 & 0.96 & 0.98 & 0.95 & 0.91 \\
$b_{11}$ & 31 & 38 & 34 & 29 & 31 & 38 & 34 & 29 & 31 & 38 & 34 & 29 \\
$b_{12}$ & 0.95 & 0.93 & 0.92 & 0.96 & 0.92 & 0.97 & 0.94 & 0.90 & 0.93 & 0.95 & 0.96 & 0.94 \\
\hline
\end{tabular}

\subsection{Calculations and results}

Step 1: Normalize decision matrices provided by $D M_{1}, D M_{2}, D M_{3}$ with Equation (3);

Step 2: Calculate the CGRA degree of each alternative under $D M_{1}, D M_{2}, D M_{3}$;

Considering that there is no obvious preference for the three kinds of relational degrees, let $w_{1}=w_{2}=w_{3}=1 / 3$. The CGRA degree $\phi_{i}^{(k)}$ of each alternative $a_{i}(i=1,2,3,4)$ under each decision maker $D M_{k}(k=1,2,3)$ can be calculated by Equations (4)-(13), that are

$$
\begin{aligned}
& \phi_{1}^{(1)}=0.7413, \phi_{2}^{(1)}=0.9189, \phi_{3}^{(1)}=0.8896, \phi_{4}^{(1)}=0.5507 ; \phi_{1}^{(2)}=0.6113, \phi_{2}^{(2)}=0.9775, \\
& \phi_{3}^{(2)}=0.8829, \phi_{4}^{(2)}=0.5287 ; \phi_{1}^{(3)}=0.6061, \phi_{2}^{(3)}=0.9535, \phi_{3}^{(3)}=0.9254, \phi_{4}^{(3)}=0.5340 .
\end{aligned}
$$

Step 3: Construct the information DOI of $D M_{1}, D M_{2}, D M_{3}$ and the Mass functions of the alternatives and the overall uncertainty under $D M_{1}, D M_{2}, D M_{3}$;

The information DOI of each $D M_{k}(k=1,2,3)$ can be calculated by Equation (14), namely, $\quad D O I\left(D M_{1}\right)=0.760043, D O I\left(D M_{2}\right)=0.726746, D O I\left(D M_{3}\right)=0.731031$. The Mass functions of the alternatives and the overall uncertainty under $D M_{1}, D M_{2}, D M_{3}$ are as follows: $m_{1}^{(1)}=0.1779, m_{2}^{(1)}=0.2205, m_{3}^{(1)}=0.2135$, $m_{4}^{(1)}=0.1321 \quad ; \quad m_{1}^{(2)}=0.1670 \quad, \quad m_{2}^{(2)}=0.2671 \quad, \quad m_{3}^{(2)}=0.2412 \quad, \quad m_{4}^{(2)}=0.1445 \quad ;$ $m_{1}^{(3)}=0.1630, m_{2}^{(3)}=0.2565, m_{3}^{(3)}=0.2489, m_{4}^{(3)}=0.1436 \quad . \quad m_{\Theta}^{(1)}=0.256024$, 
$m_{\Theta}^{(2)}=0.180119, m_{\Theta}^{(3)}=0.187978$

Step 4: Calculate the integrated Mass functions;

Firstly, let the weight vector of $D M_{1}, D M_{2}, D M_{3}$ be $\psi=(0.275,0.318,0.407)$, the modified Mass functions of $a_{i}(i=1,2,3,4)$ and the overall uncertainty are as follows: $\hat{m}_{1}^{(1)}=0.1202, \hat{m}_{2}^{(1)}=0.1490, \hat{m}_{3}^{(1)}=0.1442, \hat{m}_{4}^{(1)}=0.0893 ; \hat{m}_{1}^{(2)}=0.1305$, $\hat{m}_{2}^{(2)}=0.2087, \hat{m}_{3}^{(2)}=0.1885, \hat{m}_{4}^{(2)}=0.1129 ; \hat{m}_{1}^{(3)}=0.1630 \quad, \hat{m}_{2}^{(3)}=0.2565$, $\hat{m}_{3}^{(3)}=0.2489, \hat{m}_{4}^{(3)}=0.1436 . \hat{m}_{\Theta}^{(1)}=0.4973, \hat{m}_{\Theta}^{(2)}=0.3594, \hat{m}_{\Theta}^{(3)}=0.1880$.

Then, according to Dempster's combination rule, the integrated Mass functions of $a_{i}(i=1,2,3,4)$ and the overall uncertainty are as follows: $m\left(a_{1}\right)=0.1753$, $m\left(a_{2}\right)=0.3130, m\left(a_{3}\right)=0.2897, m\left(a_{4}\right)=0.1408 ; m(\Theta)=0.0812$

Step 5: Calculate the Pignistic probability value of each alternative;

The Pignistic probability values of alternatives are as follows: $\operatorname{Bet} P\left(a_{1}\right)=0.1956$, $\operatorname{Bet} P\left(a_{2}\right)=0.3333, \operatorname{Bet} P\left(a_{3}\right)=0.3100, \operatorname{Bet} P\left(a_{4}\right)=0.1610$.

Step 6: Rank alternatives.

According to the Pignistic probability values, the final ranking of alternative strategies is obtained as follows: $a_{2} \mathrm{f} a_{3} \mathrm{f} a_{1} \mathrm{f} a_{4}$, that is, $a_{2}$ is the best, and the enterprise may consider cooperating with supplier $a_{2}$ first.

\subsection{Comparative analysis}

In order to verify the feasibility and effectiveness of the proposed method, the above evaluation results are compared with those of other methods, as shown in Table 3.

Table 3 The Comparison Results of Relevant Methods Pignistic probability values Ranking orders Decision results 


\begin{tabular}{|c|c|c|c|}
\hline The method based on D-S & $\begin{array}{l}\operatorname{Bet} P\left(a_{1}\right)=0.1642, \operatorname{Bet} P\left(a_{2}\right)=0.3745 \\
\operatorname{Bet} P\left(a_{3}\right)=0.3518, \operatorname{Bet} P\left(a_{4}\right)=0.1963\end{array}$ & $a_{2} \mathrm{f} a_{3} \mathrm{f} a_{4} \mathrm{f} a_{1}$ & $a_{2}$ \\
\hline $\begin{array}{l}\text { The method based on classical } \\
\text { GRA }\end{array}$ & $\begin{array}{l}\operatorname{Bet} P\left(a_{1}\right)=0.2157, \operatorname{Bet} P\left(a_{2}\right)=0.4386 \\
\operatorname{Bet} P\left(a_{3}\right)=0.4624, \operatorname{Bet} P\left(a_{4}\right)=0.2031\end{array}$ & $a_{3} \mathrm{f} a_{2} \mathrm{f} a_{1} \mathrm{f} a_{4}$ & $a_{3}$ \\
\hline The proposed method & $\begin{array}{l}\operatorname{Bet} P\left(a_{1}\right)=0.1956, \operatorname{Bet} P\left(a_{2}\right)=0.3333 \\
\operatorname{Bet} P\left(a_{3}\right)=0.3100, \operatorname{Bet} P\left(a_{4}\right)=0.1610\end{array}$ & $a_{2} \mathrm{f} a_{3} \mathrm{f} a_{1} \mathrm{f} a_{4}$ & $a_{2}$ \\
\hline
\end{tabular}

As can be seen from Table 3, the ranking orders based on D-S and classical GRA are not exactly the same as that of the proposed method. The reason for the difference between the method based on D-Sand the proposed method may be that the method based on D-S does not distinguish the reliability of evidence information under individual decisionmakers, which ignores the differences among individuals. The proposed method not only considers the reliabilities of individuals, but also uses the evidence weight to modify the evidence information, which make the group decision-making fusion result more reasonable.

In addition, the reason for the different ranking results between the method based on classical GRA and the proposed method may be that the method based on classical GRA only considers the similarity of geometric shapes between the alternative sequence and the positive (negative) ideal point sequence to measure the relational degree. The proposed method uses CGRA to measure the proximity, similarity and volatility of the development and evolution between sequences comprehensively, which lays a foundation for obtaining Mass functions under individual decision makers more scientifically.

\section{Conclusions}

In this paper, a group decision making method based on GRA and evidence theory is proposed to solve the group decision making problems. Firstly, combining GRA with evidence theory, a CGRA method is proposed to determine the degree of ignorance for individual decision makers' information and the Mass function. Then, 
the weighted evidence theory is adopted to fuse the group decision-making information. On this basis, the alternatives are ranked according to Pignistic probability values. Finally, an example of green supplier selection is given to illustrate the effectiveness of the proposed method. Meanwhile, the proposed method also provides a new way to solve the group decision making problems in reality.

The paper makes three important contributions. Firstly, a CGRA method is proposed to measure the proximity, similarity, and volatility between sequences synthetically, which makes the determination of Mass function more objective. Secondly, the reliability of decision makers' evidence information is introduced into evidence theory, and the individual evidence information is modified by the evidence weight, which lays a scientific basis for subsequent reasoning fusion decision-making. Thirdly, on the basis of considering the overall uncertainty of the system, the Mass function is transformed into probability by Pignistic probability transformation, which effectively reduces the information loss during the transformation.

In this paper, the group decision making problem is studied from the perspective of static information fusion, and the dynamic reasoning decision-making problem that the generation of reasoning information may require interactive discussions among decisionmakers is not considered. Hence, expanding this problem from the perspective of dynamic interaction is the focus of the next research. Additionally, the weights of proximity, similarity and volatility in CGRA degree are mainly determined subjectively, and the quantitative optimization method can be further studied to determine the weights objectively.

\section{Acknowledgments}

The authors are grateful to the anonymous referees for useful suggestions that have improved the quality of this paper. This research is supported by the National Natural 
Science Foundation of China (51979106), Scientific and Technological Plan Fund Project of Henan Province (182102310014), Key Research Project Plan of Henan Universities (18A630030, 18A630032), and the Quality Curriculum of Postgraduate Education in Henan Province (HNYJS2015KC02).

Conflict of interest: The authors declare that they have no conflict of interest.

Data availability: The data that support the findings of this paper are available from the corresponding author.

\section{References}

Amin S.H., Zhang G.Q., An integrated model for closed-loop supply chain configuration and supplier selection: Multi-objective approach, Expert Systems with Applications, (2012), 39(8): 6782-6791.

Azadi M., Jafarian M., Saen R.F., Mirhedayatian S.M., A new fuzzy DEA model for evaluation of efficiency and effectiveness of suppliers in sustainable supply chain management context, Computers\& Operations Research, (2015), (54): 274-285.

Cali S., Balaman S.Y., A novel outranking based multi criteria group decision making methodology integrating ELECTRE and VIKOR under intuitionistic fuzzy environment,Expert Systems with Applications, (2019), 119:36-50.

Chemoun K., Gilg M., Laghrouche M., Lorenz P., Evidence theory-based framework for improving automation in home automation system, International Journal of Communication Systems, (2018),31(17): e3791.

Govindan K., Kadzi' nski M., Sivakumar R., Application of a novel PROMETHEE-based method for construction of a group compromise ranking to prioritization of green suppliers in food supply chain, Omega, (2017), 71: 129-145.

Govindan K., Kaliyan M., Kannan D., Haq A.N., Barriers analysis for green supply chain management implementation in Indian industries using analytic hierarchy process, International Journal of Production Economics, (2014), (147): 555-568.

Gupta P., Mehlawat M.K., Grover N., Intuitionistic fuzzy multi-attribute group decision-making with an application to plant location selection based on a new extended VIKOR method. Information Sciences, (2016), (370):184-203. 
Hamdan S., Cheaitou A., Supplier selection and order allocation with green criteria: An MCDM and multi-objective optimization approach, Computers \& Operations Research, (2017), (81): 282-304.

Khojasteh M., Jadid S., Stochastic risk-constraint pricing strategy of electricity retailers based on Dempster-Shafer evidence theory, Energy Strategy Reviews, (2017), (18):260-274.

Leung Y., Ji N.N., Ma J.H., An integrated information fusion approach based on the theory of evidence and group decision-making, Information Fusion, (2013),14(4):410-422.

Li X.M., Hipel K.W., Dang Y.G., An improved grey relational analysis approach for panel data clustering, Expert Systems with Applications, (2015),42(23):9105-9116.

Lin C.H., Madu C.N., Kuei C.H., Tsai H.L., Wang K.N., Developing an assessment framework for managing sustainability programs: A Analytic Network Process approach, Expert Systems with Applications, (2015), 42(5): 2488-2501.

Lin Y., Li Y.Y., Yin X.H., Dou Z., Multisensor Fault Diagnosis Modeling Based on the Evidence Theory. IEEE Transactions on Rellability, (2018),67(2):513-521.

Liu Z., Dang Y.G., Zhou W.J., Xia W.G., New grey nearness incidence model and its extension, Control and Decision, (2014),29(6):1071-1075. [In Chinese]

Luthra S., Govindan K., Kannan D., Mangla S.K., Garg C.P., An integrated framework for sustainable supplier selection and evaluation in supply chains, Journal of Cleaner Production,(2017), (140):1686-1698.

Mafakheri F., Breton M., Ghoniem A., Supplier selection-order allocation: A two-stage multiple criteria dynamic programming approach, International Journal of Production Economics, (2011),132(1):52-57.

Peng K. K., Risk Evaluation for Bridge Engineering Based on Cloud-Clustering group decision method, Journal of Performance of Constructed Facilities, (2019), 33(1):04018105.

Qin J.D., Liu X.W., Pedrycz W., An extended TODIM multi-criteria group decision making method for green supplier selection in interval type-2 fuzzy environment, European Journal of Operational Research, (2017), 258(2): 626-638.

Roshandel J., Miri-Nargesi S.S., Hatami-Shirkouhi L., Evaluating and selecting the supplier in detergent production industry using hierarchical fuzzy TOPSIS, Applied Mathematical Modelling, (2013), 37(24): 10170-10181.

Seiti H., Hafezalkotob A., Developing pessimistic-optimistic risk-based methods for 
multi-sensor fusion: An interval-valued evidence theory approach. Applied Soft Computing, (2018), (72):609-623.

Smets P., Decision making in the TBM: the necessity of the pignistic transformation. International Journal of Approximate Reasoning, (2005),38(2):133-147.

Sun H.F., Dang Y.G., Mao W.X., A Decision-Making Method with Grey Multi-Source Heterogeneous Data and Its Application in Green Supplier Selection. International Journal of Environmental Research and Public Health, (2018),15(3):446.

Tang J., Meng F.Y., Zhang S.L., An Q.X., Group decision making with interval linguistic hesitant fuzzy preference relations, Expert Systems with Applications, (2019), 119: 231-246.

Tsui C.W., Wen U.P., A Hybrid Multiple Criteria Group Decision-Making Approach for Green Supplier Selection in the TFT-LCD Industry, Mathematical Problems in Engineering, (2014): 1-13.

Wu T., Blackhurst J., Supplier evaluation and selection: An augmented DEA approach, International Journal of Production Research, (2009), 47(16): 4593-4608.

Wan S.P., Wang Q.Y., Dong J.Y., The extended VIKOR method for multi-attribute group decision making with triangular intuitionistic fuzzy numbers. Knowledge-based Systems, (2013), (52):65-77.

Wang H. F., Lin D.Y., Qiu J., Ao L.L., Du Z.D., He B.T., Research on Multiobjective Group Decision-Making in Condition-Based Maintenance for Transmission and Transformation Equipment Based on D-S Evidence Theory, IEEE Transactionson Smart Grid, (2015),6(2):1035-1045.

Wang Y.H., Dang Y.G., Approach to interval numbers investment decision-making based on grey incidence coefficients and D-S theory of evidence, Systems Engineering-Theory \&Practice, (2009),29(11):128-134.

Wang Z.X., Yao P.Y., Grey Relational Analysis of Economic Policy Uncertainty in Selected European Union Countries, Economic Computationand Economic Cybernetics Studies and Research, (2018),52(2):251-265.

Zhou Y.H., Tao X., Luan L., Wang Z.H., Safety justification of train movement dynamic processes using evidence theory and reference models. Knowledge-based Systems, (2018), (139):78-88. 